\title{
Enhancement of the Protein Loading Density by a Pre-Cleaning Process of a Gold Substrate: Confocal Laser Scanning Microscopic Study
}

\author{
Taehan Park,* Jaebum Choo, ${ }^{* \dagger}$ Moonkwon Lee, ${ }^{*}$ Yang S. Kim,**† Eun Kyu LeE,** \\ and Hae Seong LEE***
}

\author{
*Department of Applied Chemistry, Hanyang University, Ansan 426-791, South Korea \\ **Micro Biochip Center, Hanyang University, Ansan 426-791, South Korea \\ ***Korea Basic Science Institute, Jeonju 561-756, South Korea
}

\begin{abstract}
The immobilization of glucose oxidase (GOx), using self assembled monolayers (SAMs) on gold surfaces, was investigated by grazing angle FT-IR spectroscopy, surface plasmon resonance (SPR) spectroscopy, and atomic force microscopy (AFM) in conjunction with confocal laser scanning microscopy (CLSM). To find an optimum condition for the maximum GOx loading density on gold surfaces, different cleaning protocols were examined. The loading density of GOx on surfaces was investigated by AFM and CLSM. In particular, CLSM was more effective for identifying the GOx density than AFM, since its scanning speed is much faster and it covers a larger area. Based on CLSM images of the GOx immobilized on the surfaces, it was concluded that the pre-cleaning process of gold substrates using different solvents, such as acetone, ethanol and 2-propanol, is very important for enhancing the GOx loading density. This result enables us to investigate an effective fabrication process in fabricating biosensors.
\end{abstract}

(Received May 6, 2004; Accepted June 24, 2004)

\section{Introduction}

The immobilization of proteins using self-assembled monolayers (SAMs) on metal surfaces has been widely studied in elucidating protein interactions on surfaces and in developing biosensors, such as protein chips or enzyme electrodes, owing to a number of advantages of SAMs. ${ }^{1-8}$ They not only provide a high degree of reproducibility, ${ }^{9,10}$ but also make it possible to immobilize proteins close to the surface of an electrode for the purpose of obtaining a direct electron transfer. ${ }^{11-18}$ When the protein immobilization process using SAMs is practically applied to a biosensor, the density of loaded proteins should be greatly enhanced to improve its detection sensitivity. It was known that the ratio of alcohol-terminated $(-\mathrm{OH})$ thiol to carboxylic acid-terminated $(-\mathrm{COOH})$ thiol has an important effect on the loading density of the immobilized proteins. ${ }^{1,4}$ However, it was also found that the pre-cleaning process of gold substrates strongly affects the loading density of GOx. In the present work, different cleaning protocols of gold surfaces were examined to find the optimal conditions for the maximum GOx loading density.

To find fundamental physicochemical characteristics for the interactions between the GOx and gold surfaces, the grazing angle FT-IR and SPR spectroscopic methods were employed. In order to better understand how different cleaning protocols affect the loading density of GOx on gold surfaces, tapping mode AFM and CLSM were utilized. Tapping mode AFM is a most suitable technique for the visualization of proteins

$\uparrow$ To whom correspondence should be addressed.

E-mail: jbchoo@ hanyang.ac.kr; yangskim@microbiochip.com immobilized on surfaces since it provides a real threedimensional morphology of the loaded enzyme at a molecular level. However, it generally encounters two limitations for measuring surface morphology. First, it is difficult to visualize the overall surface coverage for an area as large as a few hundred micrometers. Second, AFM requires some effort to obtain a high-resolution image, since stability in a biological sample should be maintained during relatively long scanning periods. To resolve this problem, the CLSM technique was utilized in this work. CLSM is a very attractive tool for exploring the process of protein immobilization on the micrometer scale with a high scanning speed. In the present study, we report on the pre-cleaning process of gold substrates to enhance the GOx loading density and its fast image-viewing CLSM technique to control the optimum loading conditions.

\section{Experimental}

\section{Materials}

The following materials and chemicals were used as received: 90\% 16-mercaptohexadecanoic acid (MHA), 97\% 11-mercapto1-undecanol (MUO), 97\% N-hydroxy succinimide, 1-ethyl-3(3-(dimethylamino)propyl)carbodiimide, glucose oxidase, and fluorescein isothiocyanate (FITC). All of the solvents mentioned above were of HPLC grade, and the other reagents were of analytical grade. The solvents and reagents were purchased from either Aldrich Chemicals, Sigma Chemicals, or Molecular probes.

\section{Preparation of substrates and monolayer formation}

Gold films (100 nm thick) were prepared by the chemical 


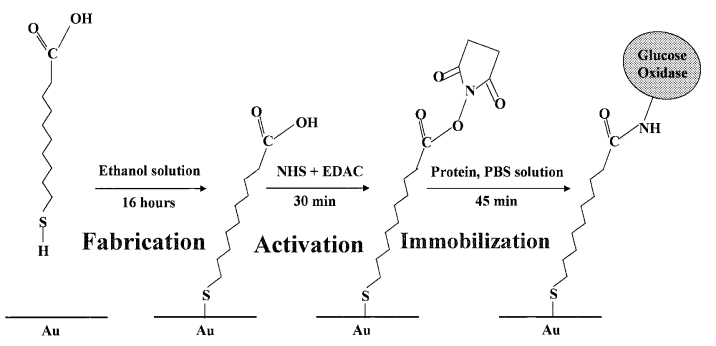

Fig. 1 Schematic representation of GOx immobilization on gold surfaces.

vapor deposition of gold (99.9\%) onto a cleaned four-inch $\mathrm{Si}$ wafer. A 50-nm-thick layer of titanium was used as an adhesive substrate. The gold films were cut into $1.0 \times 0.7 \mathrm{~cm}^{2}$ sections and were washed by three different cleaning protocols. The gold films were (a) directly placed in a piranha (hydrogen peroxide/sulfuric acid, 1:3 v/v) solution for $5 \mathrm{~min}$; (b) placed in a heated piranha solution up to $70^{\circ} \mathrm{C}$ for $5 \mathrm{~min}$; (c) washed with acetone for $10 \mathrm{~min}$ and rinsed with ethanol for $2 \mathrm{~min}$ followed by 2-propanol for $2 \mathrm{~min}$. Then all of the surfaces were thoroughly rinsed with deionized water, and dried under a stream of high-purity nitrogen gas. To form alkanethiol SAMs on the gold surfaces, these samples were immersed in $10 \mathrm{mM}$ tetrahydrofuran (THF) solutions of 11-MUO, 16-MHA (10:1) for $24 \mathrm{~h}$ before being rinsed in THF, followed by deionized water. The samples were then submerged in a solution of 150 $\mathrm{mM}$ EDAC and $30 \mathrm{mM}$ NHS for $30 \mathrm{~min}$ and rinsed with deionized water to remove any material that had not become attached to the gold surfaces. The samples were then dried under a nitrogen gas purge and stored in a desiccator. In this way, the carboxylate terminated SAMs were activated for the stable immobilization of GOx.

\section{Glucose oxidase immobilization}

For GOx immobilization, SAM-modified gold surfaces were immersed in a GOx solution (266700 units/g solid, 80\% protein). The GOx was expected to be immobilized on gold surfaces by covalent bonding. For this purpose, surface that had been modified with SAMs was treated with a stirred 0.1 M PBS buffer solution at $\mathrm{pH}$ 5.5. The buffer solution also contained 30 $\mathrm{mM}$ NHS and $150 \mathrm{mM}$ EDC. After $30 \mathrm{~min}$, the surface was rinsed with phosphate buffer and immediately placed in a stirred solution of $2 \mathrm{mg} / \mathrm{mL}$ GOx with $0.1 \mathrm{M}$ sodium phosphate buffer at $\mathrm{pH}$ 5.5. This step was allowed to proceed overnight at room temperature. The surface was then thoroughly rinsed with deionized water. Carboxylate-terminated SAMs are usually used to achieve a stable immobilization of proteins by covalent bonding in the esterification of $N$-hydroxy succinimide (NHS) with water-soluble 1-ethyl-3-(3-(dimethylamino)propyl) carbodiimide (EDC). Immobilization occurs by displacing the NHS group by the lysine residues of the protein, as shown in Fig. 1. There has been considerable research carried out into the time required to achieve the stable formation of SAMs, ${ }^{19-21}$ and on the optimum conditions for activating carboxylic acidterminated SAMs with EDC and NHS. ${ }^{22-24}$

\section{Instrumentation}

\section{Grazing angle FT-IR}

All of the IR spectra were collected using a Bio-Rad FTS 6000 FT-IR spectrometer equipped with a $\mathrm{KBr}$ beam splitter
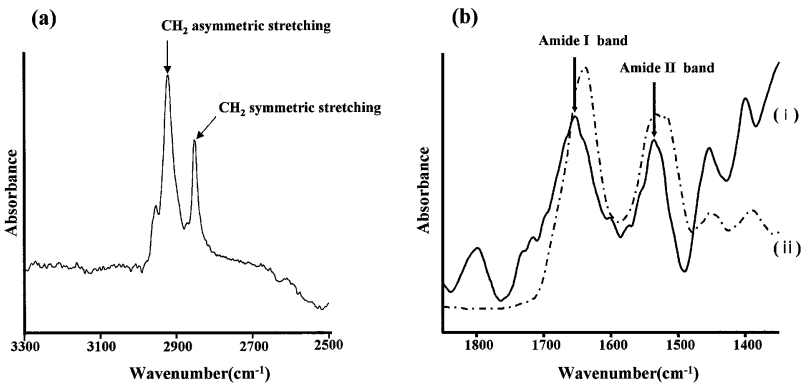

Fig. 2 (a) Grazing-angle FT-IR spectrum showing the $-\mathrm{CH}$ stretch region for a $1: 1$ mixture of $\mathrm{C} 16 \mathrm{COOH} / \mathrm{C} 11 \mathrm{OH} \mathrm{SAM}$ on gold surfaces. (b) Comparison of (i) the grazing angle FT-IR spectrum of immobilized GOx on a mixed $\mathrm{C} 16 \mathrm{COOH} / \mathrm{C} 11 \mathrm{OH}$ SAM, and (ii) the absorbance FT-IR spectrum of pure GOx.

and a MCT detector. The grazing-angle FT-IR spectra of the SAMs on gold surfaces and the GOx immobilized on the SAMs were recorded using a grazing-angle sample compartment. To increase the signal-to-noise ratio, 1024 scans were collected at a resolution of $1.0 \mathrm{~cm}^{-1}$.

\section{Surface plasmon resonance}

The SPR spectra were measured using a BIAcore 300 system. SPR spectroscopy allows the acquisition of kinetic data of protein on surfaces in real time by obtaining changes in the refraction index of the layer. $1,3,8,25$ It can provide useful information about the adsorption rate and equilibrium constant of protein interactions on gold surfaces. This technique also provides information on the amount of proteins bound to the surface. This is a very strong advantage of SPR spectroscopy, since there are few analytical techniques to provide quantitative information of immobilized proteins on surfaces.

\section{Atomic force microscopy}

AFM images of the GOx samples were obtained using a Nanoscope IV (Digital Instruments Inc., Santa Barbara, USA) under the tapping imaging mode. Images were acquired using a silicon cantilever unit at a scan rate of $1.5 \mathrm{~Hz}$. A sharpened silicon nitride tip (force constant: $4 \mathrm{~N} / \mathrm{m}$, Mikro Masch, Russia) was used. The drive amplitude range was between 0.7 and 0.75 $\mathrm{mV}$. The protein dimensions, such as the height and size, were determined using section analysis in Nanoscope IV software tools.

\section{Confocal laser scanning microscopy}

GOx fluorescence images of gold surfaces were obtained using a Leica TCS SP inverted CLSM equipped with four photomultiplier tube (PMT) detectors with a pixel size of $0.48 \times$ $0.48 \mathrm{~m}^{2}$. Before a measurement, the GOx was labeled with an FITC fluorescence tag. First, $10 \mathrm{mg}$ of GOx was dissolved in 1 $\mathrm{mL}$ of 0.1 M PBS buffer solution (pH 5.5). Secondly, $2 \mathrm{mg}$ of FITC dye was dissolved in $1 \mathrm{~mL}$ of DMSO. While vortexing the GOx solution in step $1,100 \mu \mathrm{L}$ of the reactive FITC dye was slowly added. This reaction was incubated for $1 \mathrm{~h}$ at room temperature with continuous stirring. Finally, the unreacted labeling FITC reagent was separated from the conjugated proteins using centrifugal filter devices.

The laser provided an FITC probe excitation wavelength of $\lambda=488 \mathrm{~nm}$, and the emitted fluorescence light was detected at $\lambda=510 \mathrm{~nm}$. Individual images of GOx immobilized on gold surfaces were analyzed by horizontal scanning of the twodimensional confocal images in the $x-y$ plane perpendicular to 


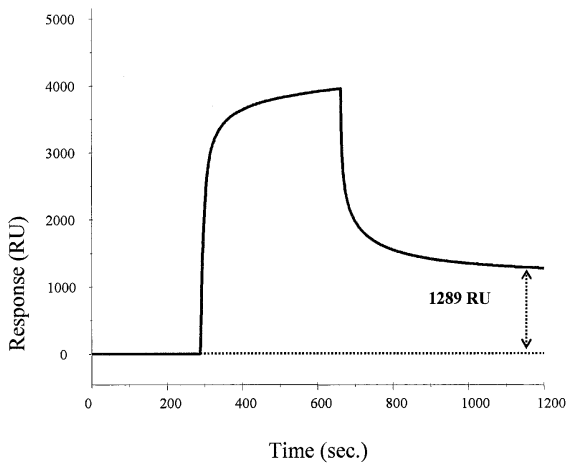

Fig. 3 SPR spectrum of FITC-tagged GOx immobilized on gold surfaces. The GOx concentration was $0.5 \mu \mathrm{g} / \mu \mathrm{L}$ in $10 \mathrm{mM}$ phosphate buffer at $\mathrm{pH}=7$.

the optical axis. To reduce any background fluorescence and noise, all of the images were generated by accumulating four scans per image. Topographic images and fluorescence signals could be simultaneously detected using the CLSM.

\section{Results and Discussion}

\section{Grazing angle FT-IR}

Grazing-angle FT-IR spectra were measured to confirm the formation of SAMs as well as the immobilization of GOx on the gold surfaces. Figure 2(a) shows the grazing angle FT-IR spectra of a mixed SAM with 11-MUO/16-MHA in a ratio of 10:1 on gold surfaces. Three different types of $-\mathrm{CH}$ stretch bands were observed in the region of $2920-2850 \mathrm{~cm}^{-1}$. These of vibrational bands were used to identify the formation of SAMs on the gold surfaces. On the other hand, the successful attachment of GOx on the SAM-modified gold surfaces was confirmed by the formation of characteristic amide bands, as shown in Fig. 2(b). The amide I and II bands of GOx appeared at 1680 and $1550 \mathrm{~cm}^{-1}$, respectively. For comparison purposes, the absorbance infrared spectrum of pure GOx was also measured, and is also shown in Fig. 2(b). Even though there is a small band shift due to conformational changes during the immobilization process, ${ }^{8}$ the band positions and intensities of the two different GOx samples show a good one-to-one correspondence, as can be seen from Fig. 2(b). Thus, it can be concluded that the GOx had become successfully attached to the SAM-modified gold surface.

\section{Surface plasmon resonance spectroscopy}

Figure 3 shows the SPR data for the adsorption of GOx on mixed SAMs. The concentration of GOx was $0.5 \mu \mathrm{g} / \mu \mathrm{L}$ in 10 $\mathrm{mM}$ phosphate buffer at $\mathrm{pH}$ 7. The angle difference, expressed in resonance signal, was 1289 RU. Assuming that the molecular weight of GOx is $\sim 160000$, the number of GOx molecules was calculated to be about $3.76 \times 10^{9}$. Since the molecular size of GOx in the $x y$-plane is approximately $13 \mathrm{~nm} \times$ $5.2 \mathrm{~nm}$, this value means that $20 \%$ of the surfaces was covered by the GOx.

\section{Atomic force microscopy}

AFM images were obtained to provide a nanoscopic view of immobilized GOx on the gold surfaces. The surface topographies were recorded using a scan rate of $1.5 \mathrm{~Hz}$ in a scanned area of $500-1000 \mathrm{~nm}$. After the immobilization of GOx on gold surfaces, we attempted to visualize the molecular
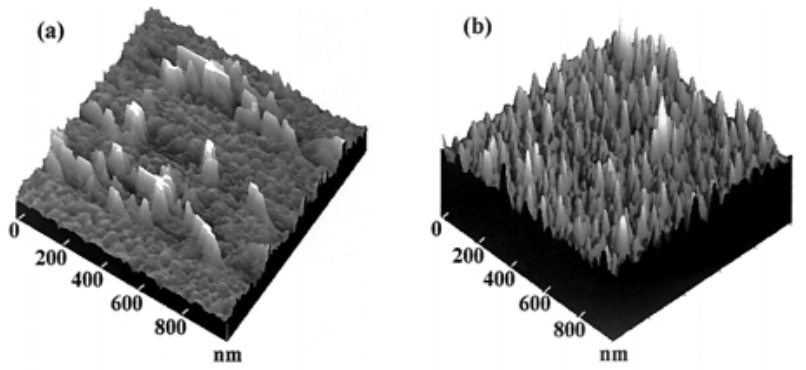

Fig. 4 (a) AFM image for a low-loading GOx density immobilized on a mixed $\mathrm{C} 16 \mathrm{COOH} / \mathrm{C} 11 \mathrm{OH}$ SAM (cleaning protocol (a)). (b) AFM image of a high-loading GOx density immobilized on a mixed $\mathrm{C} 16 \mathrm{COOH} / \mathrm{C} 11 \mathrm{OH}$ SAM (cleaning protocol (c)).

shapes of GOx using the AFM. It was very important to observe a well-defined morphological image on the surface, since our goal was to determine the optimum conditions to increase the loading density of GOx on the gold surfaces.

Figure 4(a) shows a three-dimensional AFM image of an immobilized low loading GOx density on a SAM. In this case, the gold films were directly placed in a piranha solution for 5 min before the immobilization of the GOx (cleaning protocol (a)). The loading density of the GOx could be improved by treating the surfaces with acetone, ethanol, and 2-proponal before being placed it in a pirana solution. Figure 4(b) shows a three-dimensional AFM image of a high loading GOx density on surfaces. However, it was very inefficient to have to determine the optimum cleaning conditions using the AFM, since it took a very long time to obtain an image for each cleaning condition. As previously mentioned, the CLSM technique was therefore utilized to quickly determine the optimum cleaning condition.

\section{Confocal laser scanning microscopy}

The loading density of immobilized proteins on the mixed SAMs is sensitive to slight changes in the processing conditions. Due to a fast laser scanning speed, CLSM took just a few minutes to obtain a fluorescence image for the immobilized GOx using this technique. Figure 5 shows three different laser scanning fluorescence images for an immobilized GOx on SAMs employing different initial substrate cleaning conditions. This figure shows that the coverage of GOx is quite different for each of three samples, even though they showed very similar grazing-angle FT-IR spectra. The amount of immobilized GOx was very small in the case of cleaning with the piranha solution for a period of $5 \mathrm{~min}$ (protocol (a)). In the case of cleaning using protocol (b), an aggregation of GOx was observed over the entire surface. For cleaning using protocol (c), the cluster size increased further, which means that the amount of immobilized GOx had increased. These results were reproducible over many repeated experiments and demonstrate that CLSM is a very effective image-scanning method for the fast identification of protein immobilization.

\section{Conclusion}

The immobilization of GOx on gold surfaces has been investigated using grazing-angle FT-IR, SPR, AFM and CLSM techniques. The FT-IR spectra of the GOx-immobilized surfaces showed the presence of amide I and II bands, which confirmed that the GOx was successfully immobilized on the 


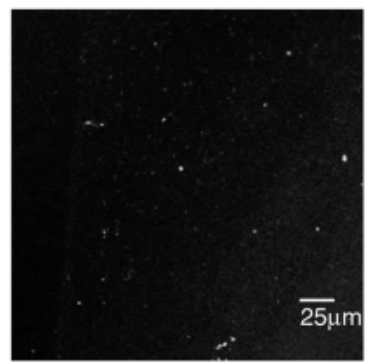

(a)

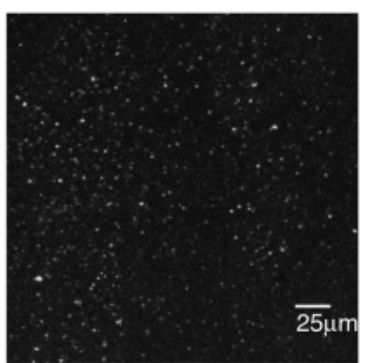

(b)

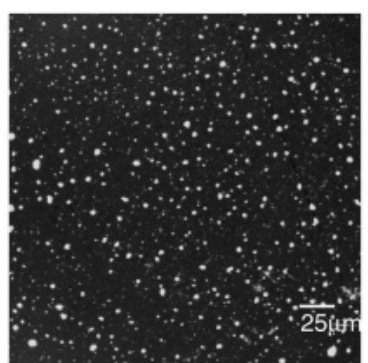

(c)

Fig. 5 Confocal laser scanning fluorescence images of immobilized GOx on SAMs for three different initial substrate cleaning conditions: (a) placed in a piranha solution for $5 \mathrm{~min}$, (b) placed in a heated piranha solution up to $70^{\circ} \mathrm{C}$ for $5 \mathrm{~min}$, and (c) pretreated with acetone, ethanol, and 2-propanol before being placed in piranha solution for 5 min without heating.

gold surfaces. The SPR spectrum allowed us to figure out the surface coverage of GOx as well as the acquisition of adsorption data of GOx on the surfaces in real time. AFM and CLSM were utilized in a complimentary manner to image the GOximmobilized surfaces. Using the AFM technique, morphological information on the GOx-immobilized surfaces was obtained at the molecular level. In the immobilization of SAMs and GOx, including fabrication, activation and immobilization, it was found that the cleaning process of gold surfaces strongly affected its loading density. To find the optimum conditions for the maximum GOx loading density, different cleaning protocols had to be examined. Furthermore, a fast experimental method to image the GOx-immobilized surfaces treated with different cleaning methods was required. The CLSM technique is applicable to this problem. With the CLSM, it took just a few minutes to obtain a reliable scanned image, showing the GOx loading condition. According to the CLSM images, the GOx loading density was maximized when the gold surfaces were pretreated with acetone, ethanol, and 2propanol before being placed in a piranha solution.

\section{Acknowledgements}

This research was supported by a grant (M102KN01-000102K1401-00520) from Center for Nanoscale Mechatronics \& Manufacturing, one of 21st Centry Frontier Research Programs, Ministry of Science and Technology, Korea. J. C. thanks the Korean Science and Engineering Foundation (Grant Number R14-2002-004-01000) for financial support. H. L. thanks for the financial support from National R\&D Project for Nano Science and Technology (Contract No. M1021400011002B1500-01800) of MOST.

\section{References}

1. E. Ostuni, L. Yan, and G. M. Whitesides, Colloids Surf., B, 1999, 15,3 .

2. H. O. Finklea, "Encyclopedia of Analytical Chemistry", 2000, John Wiley \& Sons Ltd., Chichester.

3. N. Patel, M. C. Davies, M. Hartshorne, R. J. Heaton, C. J. Roberts, S. J. B. Tendler, and P. M. Williams, Langmuir, 1993, 13, 6485.

4. J. J. Gooding, P. Erokhin, D. Losic, W. Yang, V. Policarpio, J. Liu, F. M. Ho, M. Situmorang, D. B. Hibbert, and J. G. Shapter, Anal. Sci., 2001, 17, 3.
5. D. Losic, J. G. Shapter, and J. J. Gooding, Langmuir, 2002, 18,5422 .

6. D. B. Hibbert, J. J. Gooding, and P. Erokhin, Langmuir, 2002, 18, 1770 .

7. J. J. Gooding, L. Pugliano, D. B. Hibbert, and P. Erokhin Electrochem. Commun., 2000, 2, 217.

8. A. J. Guiomar, J. T. Guthri, and S. D. Evans, Langmuir, 1999, 15, 1198.

9. J. J. Gooding, V. Praig, and E. A. H. Hall, Anal. Chem., 1998, 70, 2396

10. J. J. Gooding, P. Erokhin, and D. B. Hibert, Biosens. Bioelectron., 2000, 15, 229.

11. K. T. Kinnear and H. G. Monbouquette, Langmuir, 1993, 9 , 2255.

12. T. Lotzbeyer, W. Schuhmann, E. Katz, J. Falter, and H. L. Schmidt, J. Electroanal. Chem., 1994, 377, 291.

13. T. Lotzbeyer, W. Schuhmann, E. Katz, J. Falter, and H. L. Schmidt, Sens. Actuators, 1996, B33, 50.

14. T. Lotzbeyer, W. Schuhmann, E. Katz, J. Falter, and H. L. Schmidt, Bioelectroch. Bioener., 1997, 42, 1.

15. J. Li, G. Cheng, and S. Dong, J. Electroanal. Chem., 1996, 416, 97

16. E. Katz, A. Riklin, V. Heleg-Shabtai, I. Willner, A. F. L. Buckmann, and A. Heller, J. Am. Chem. Soc., 1996, 118, 10132.

17. M. Mrksich and G. M. Whitesides, Ann. Rev. Biomol. Struct., 1996, 25, 55.

18. T. Wink, S. J. Van Zuilen, A. Bult, and W. P. Van Bennekom, Analyst, 1997, 122, 43R

19. A. Ulman, "An Introduction to Ultrathin Organic Films, from Langmuir-Blodgett to Self-assembly", 1991, Academic Press, San Diego.

20. C. D. Basin, J. Evall, and G. M. Whitesides, J. Am. Chem. Soc., 1989, 111, 7155 .

21. K. E. Nelson, L. Gamble, L. S. Jung, M. S. Boeckl, E. Naeemi, S. L. Golledge, T. Sasaki, D. G. Castner, C. T. Campbell, and P. S. Stayton, Langmuir, 2001, 17, 2807.

22. N. Patel, M. C. Davis, M. Hartshorne, R. J. Heaton, C. J. Roberts, S. J. B. Tendler, and P. M. Williams, Langmuir, 1997, 13, 6485.

23. M. Veiseh, M. H. Zareie, and M. Zhang, Langmuir, 2002 , 18,6671 .

24. M. Veiseh, Y. Zhang, K. Hinkley, and M. Zhang, Biomed. Microdev., 2001, 3, 43.

25. R. G. Chapman, E. Ostuni, S. Takayama, R. E. Holmlin, L. Yan, and G. Whitesides, J. Am. Chem. Soc., 2000, 122, 8303. 